\title{
Farnesol Decreases Biofilms of Staphylococcus epidermidis and Exhibits Synergy With Nafcillin and Vancomycin
}

\author{
MOHAN PAMMI, RONG LIANG, JOHN M. HICKS, JIM BARRISH, AND JAMES VERSALOVIC \\ Departments of Pediatrics [M.P., R.L.] and Pathology [J.M.H., J.B., J.V.], Texas Children's Hospital \& Baylor College of Medicine, \\ Houston, Texas 77030
}

\begin{abstract}
Biofilm infections are frequently caused by Staphylococcus epidermidis, are resistant to antimicrobial agents, and adversely affect patient outcomes. We evaluated farnesol (FSL), the Candida quorum-sensing molecule, on $S$. epidermidis biofilms, in vitro and in vivo. We evaluated $\mathrm{ED}_{50}, \mathrm{ED}_{75}$, and $\mathrm{ED}_{90}$ (drug concentrations causing 50\%, 75\%, and 90\% inhibition, respectively) of FSL and evaluated synergy with nafcillin and vancomycin. FSL's effects on morphology of $S$. epidermidis biofilms were analyzed using confocal microscopy and real-time changes using a bioluminescent strain of $S$. epidermidis, Xen 43. In mice, effects of FSL treatment on s.c. catheter biofilms; cultures of blood, kidney, and catheter and pericatheter tissues; and bioluminescence in strain Xen 43 were evaluated. FSL inhibited biofilms $\left(\mathrm{ED}_{50}\right.$ ranged from 0.625 to $2.5 \mathrm{mM}$ ) and was synergistic with nafcillin and vancomycin at most combination ratios. FSL significantly decreased biovolume, substratum coverage, and mean thickness of S. epidermidis biofilms. In mice, FSL significantly decreased viable colony counts of $S$. epidermidis from blood, kidney, and catheter and pericatheter tissues and decreased Xen 43 bioluminescence. We confirmed the antibiofilm effects of FSL both in vitro and in vivo, in a bioluminescent strain and its synergy with antibiotics. FSL may be effective against clinical S. epidermidis biofilm infections. (Pediatr Res 70: 578-583, 2011)
\end{abstract}

$\mathrm{B}$ iofilms are three-dimensional communities of microorganisms that are surface-associated and encased in an extracellular matrix, composed of polysaccharides, proteins, and extracellular DNA (1). Biofilms contribute significantly to medical infections that include catheter and other deviceassociated infections (2). Catheter-related blood stream infections (CRBSIs) significantly increase mortality, morbidity, and healthcare costs (3). Approximately 250,000 cases of CRBSIs occur in the United States and it costs $>\$ 50,000$ to treat an episode of CRBSI (3).

S. epidermidis is the most common etiological agent isolated in device-associated biofilm-related infections (4). S. epidermidis is a normal skin commensal, but as an opportunistic pathogen leads the list for healthcare-associated infections. Although $S$. epidermidis lacks secreted virulence factors such as the exotoxins produced by Staphylococcus aureus, it is uniquely adapted to cause chronic biofilm infections. In addition, S. epidermidis is also the major cause for late-onset

Received April 25, 2011; accepted July 7, 2011.

Correspondence: Mohan Pammi, M.D., 6621, Fannin, MC: WT 6-104, Houston, Texas 77030; e-mail: mohanv@bcm.edu

Supported by NIH (CHRCDA, K12 HD41648) [to M.P.].

The authors report no conflicts of interest. neonatal sepsis in infants born at $<1500 \mathrm{~g}$ (5). Neonatal sepsis is associated with significant mortality, morbidity, and adverse neurodevelopmental outcomes $(5,6)$.

Microbial biofilms including those of S. epidermidis are inherently resistant to host defense and antimicrobial agents and hence difficult to eradicate. Clinical treatment strategies involve treatment with multiple antibiotics and removal of the life-saving catheters and devices. Novel strategies are necessary to combat biofilm-related infections to improve clinical outcomes. Farnesol (FSL), the Candida quorum-sensing molecule, has antibiofilm activity. FSL is a sesquiterpene alcohol and is the first quorum-sensing molecule described in eukaryotes (7) and produced by most Candida species (8). Exogenous FSL inhibits the development of Candida biofilms via inhibition of filamentation (9). FSL has also been reported to have efficacy against $S$. aureus biofilms and increases biofilm susceptibility to antibiotics (10). We evaluated the antimicrobial susceptibilities of S. epidermidis biofilms to FSL and synergy with antistaphylococcal antibiotics in vitro. Quorum-sensing mutants of $S$. epidermidis form larger biofilms and are implicated in chronic biofilm infections in humans and hence we evaluated mutants of the agr and luxS quorumsensing systems in addition to clinical isolates (11). We confirmed our findings in a clinically relevant mouse model of catheter infection in vivo.

\section{METHODS}

\section{Strains and culture conditions}

Organisms. S. epidermidis strains ATCC 55133, 1457, 1457 agr mutant, 1457 luxS mutant, and Xen 43 were used. ATCC 55133 and 1457 are biofilm-forming clinical isolates. Xen 43 (Caliper Life Sciences Inc.) is a bioluminescent strain derived from 1457 and is used to monitor infection in real time (12).

Growth media. Trypticose soy agar with 5\% sheep blood was used for plating organisms and trypticose soy broth (TSB) for subcultures and antimicrobial susceptibility testing.

FSL preparation. A stock solution of trans-trans-FSL (Sigma Chemical Co.) in DMSO was freshly prepared at $500 \mathrm{mM}$ before use and diluted to required concentrations. FSL suspensions were prepared fresh to preserve the antioxidant effect. FSL was prepared in concentration ranges of 80 to 0.16

\footnotetext{
Abbreviations: $\mathbf{C E D}_{\mathbf{5 0}}$ and $\mathbf{C E D}_{\mathbf{9 0}}$, combination effective dose causing $50 \%$ and $90 \%$ inhibition, respectively; CFU/mL, colony forming units $/ \mathrm{mL}$; CI, combination index; CRBSI, catheter-related bloodstream infection; $\mathbf{E D}_{\mathbf{5 0}}$, $\mathbf{E D}_{\mathbf{7 5}}$ and $\mathbf{E D}_{\mathbf{9 0}}$, drug concentrations causing 50\%, 75\%, and 90\% inhibition, respectively; FSL, farnesol; TSB, trypticose soy broth; XTT, 2,3-bis (2methoxy-4-nitro-5-sulfophenyl)-5-[(phenyl-amino) carbonyl]-2H-tetrazolium hydroxide
} 
$\mathrm{mM}$ for biofilm susceptibility testing. Nafcillin and vancomycin were prepared in concentration ranges of 80 to $0.6 \mu \mathrm{g} / \mathrm{mL}$ for biofilm antimicrobial susceptibility testing.

Antimicrobial susceptibility testing of S. epidermidis biofilms. Biofilms were formed in 96-well microtiter plates by adding $100 \mu \mathrm{L}$ of $S$. epidermidis at approximately $10^{7}$ colony-forming units $/ \mathrm{mL}(\mathrm{CFU} / \mathrm{mL})$ in RPMI $1640(\mathrm{pH}$ of 7) and incubated for $24 \mathrm{~h}$ at $35^{\circ} \mathrm{C}$. The supernatant was discarded and the biofilms washed with PBS to remove unadhered cells. Biofilm formation was confirmed by light microscopy. Antimicrobial susceptibility of biofilms to FSL was performed in duplicate by adapting Clinical and Laboratory Standards Institute (CLSI) guidelines for planktonic antimicrobial testing (13). The $S$. epidermidis biofilms in the microtiter plates were exposed to serial dilutions of antimicrobial agent (FSL from 0.2 to $80 \mathrm{mM}$ and nafcillin and vancomycin from 80 to $0.6 \mu \mathrm{g} / \mathrm{mL}$ ) for $24 \mathrm{~h}$. The end point of inhibition of the biofilms by the antimicrobial agent was estimated by the colorimetric method using 2,3-bis (2-methoxy-4-nitro-5-sulfophenyl)-5-[(phenyl-amino) carbonyl]-2H-tetrazolium hydroxide (XTT) (14). A solution of XTT $(0.5 \mathrm{~g} / \mathrm{L})$ was prepared in PBS, filter sterilized $(0.22 \mu \mathrm{m}$ size $)$, and stored at $-80^{\circ} \mathrm{C}$. Menadione was prepared as $10 \mathrm{mM}$ solution in acetone (99.9\% HPLC grade) and stored at $-80^{\circ} \mathrm{C}$ and $100 \mu \mathrm{L}$ of which added to $100 \mu \mathrm{L}$ of XTT gives 1 $\mu \mathrm{M}$ concentration. Hundred microliters of this combination was added to the wells of 96-well microtiter plates with the biofilms with the antimicrobial and then incubated in the dark for $2 \mathrm{~h}$. Microtiter plates were centrifuged for $5 \mathrm{~min}$ at $3000 \mathrm{rpm} ; 100 \mu \mathrm{L}$ supernatant was transferred to a new microtiter plate and the color read at OD $490 \mathrm{~nm}$ (15). Growth and sterility controls on the same microtiter plate were used for comparison. The experiments were done in duplicate, repeated on 3 different days, and the mean of the readings of XTT reduction was used to determine the biofilm inhibitory concentrations. We evaluated $\mathrm{ED}_{50}, \mathrm{ED}_{75}$, and $\mathrm{ED}_{90}$ (drug concentrations causing $50 \%, 75 \%$, and $90 \%$ inhibition, respectively) of FSL, nafcillin, and vancomycin.

Confocal imaging to assess biofilm morphology. Biofilms of S. epidermidis were formed on optical bottom microwell Petri dishes (Mattek Corp.) for $24 \mathrm{~h}$ and exposed to $0.5 \mathrm{mM}$ of FSL or DMSO control for another $24 \mathrm{~h}$. The biofilms were washed with PBS, stained with LIVE/DEAD stain (Molecular probes), and examined using Zeiss Meta confocal microscope. Serial sections of the biofilm were obtained at $1-\mu \mathrm{m}$ intervals along the $z$ axis, and the $z$-stack images were analyzed with the software PHLIP, for MATLAB toolbox, biovolume (in $\mu \mathrm{m}^{3}$ ), substratum coverage (in \%), and mean thickness (in $\mu \mathrm{m})$ (16). At least two representative fields in the biofilm on any particular day were analyzed and measurements were averaged. Biofilm experiments were repeated on 3 different days. Biofilms exposed to FSL were compared with the controls by $t$ test, and statistical significance was assumed at $p<0.05$

Synergy evaluation of FSL antibiotic combinations. Biofilm inhibition by combinations of FSL with nafcillin or vancomycin was evaluated in an $8 \times$ 8 checkerboard format, in 96-well microtiter plates, in 2-fold serial dilutions across rows and columns, and inhibitory end points were assessed by the XTT reduction assay. Inhibitory effects at equipotent drug-dose ratios (1:1 ratios of $\mathrm{ED}_{50}, \mathrm{ED}_{75}$, and $\left.\mathrm{ED}_{90}\right)$ and nonequipotent ratios $(1: 2,1: 4$, or $2: 1)$ of the combinations were determined. The median effects method described by Chou (17) was used to study interactions in drug combinations by the calculation of combination indices (CIs). A CI $<1$ indicates synergy, CI $>1$ antagonism, and $\mathrm{CI}=1$ an additive effect. Multiple drug dose-effect calculations were performed using Calcusyn software (Biosoft, Cambridge) with constant ratios of drug combinations.

Mouse model of s.c. catheter infection. In 3-wk-old FVB albino mice (Charles River Laboratories Inc.) mice, 1.5 inch segment of 18-gauge Teflon catheter was inserted s.c. on the back of each animal and each animal received one catheter segment. Before insertion, catheters were immersed in suspensions of S. epidermidis $\left(10^{7} \mathrm{CFU} / \mathrm{mL}\right.$ ) for $2 \mathrm{~h}$ (catheter $\mathrm{CFU} / \mathrm{mL}$ preinsertion was 3 to $\left.510^{5} \mathrm{CFU} / \mathrm{mL}\right)$, to facilitate biofilm formation. FSL $(100 \mu \mathrm{g} / \mathrm{g}$ in 0.1 $\mathrm{mL}$ DMSO, $6.7 \mathrm{mM}, n=7$ ) or DMSO (control, $n=5$ ) was injected for 6 consecutive days from d 2 of infection, once per day, s.c. near the catheter. The dose of FSL was extrapolated from other animal studies and was higher than our estimated $\mathrm{ED}_{50}$ in vitro (18). The animals were euthanized on $\mathrm{d} 8$, and cultures of blood, kidney homogenates, and catheter (by sonication) and pericatheter (skin and s.c.) tissues were plated in serial dilutions. Catheter biofilms were confirmed by confocal and electron microscopy of explanted catheter segments. Sample size calculations revealed that a sample size of five in each group gave a power of $93 \%$ in detecting a $\log$ difference in $\mathrm{CFU} / \mathrm{mL}$ of catheter cultures. The differences in CFU/mL between FSL and DMSO treatment were assessed for significance by the Kruskal-Wallis test. The protocol for animal experiments was approved by the Institutional Animal Care and Use Committee at the Baylor College of Medicine.

Scanning electron microscopy. A 5-mm sample was cut from each of the explanted catheter segments from mice with s.c. catheter infection. The catheter samples were cut in cross-sections and fixed with $2 \%$ glutaraldehyde, followed by fixing with osmium tetroxide, tannic acid, and uranyl acetate. Fixation was followed by a series of ethanol dehydration steps, and samples were sputter-coated with gold palladium. The samples were then scanned by pathologists who were blinded for FSL treatment.

Real-time imaging using the bioluminescent strain Xen 43

In vitro. Biofilms of Xen 43 were developed in 30 wells of an opaque 96-well microtiter plate (Corning) and washed with PBS. At $48 \mathrm{~h}$, three groups of 10 wells each were exposed to DMSO, FSL ( $0.5 \mathrm{mM})$, or TSB for $24 \mathrm{~h}$. Bioluminescence was monitored at 24, 48, 72, and $96 \mathrm{~h}$ and compared among the three exposures, and the experiments were performed in duplicate. In vivo, s.c. catheter infection was also established with the bioluminescent strain Xen 43, as described earlier. s.c. injections of FSL (100 $\mu \mathrm{g} / \mathrm{g}$ in $0.1 \mathrm{~mL}$ DMSO, $6.7 \mathrm{mM}, n=5)$ or DMSO $(n=5)$ were administered from $\mathrm{d} 2$ to 5 . Live animal imaging for bioluminescence was performed daily for $5 \mathrm{~d}$.

\section{RESULTS}

Antimicrobial susceptibilities of S. epidermidis biofilms. The antimicrobial susceptibilities of $S$. epidermidis biofilms at $\mathrm{ED}_{50}, \mathrm{ED}_{75}$, and $\mathrm{ED}_{90}$ for strains ATCC 55133, 1457, 1457 agr mutant, and 1457 luxS mutant are shown in Table 1. We were unable to establish $\mathrm{ED}_{90}$ of FSL, nafcillin, or vancomycin for strains 1457, 1457 agr mutant, or the 1457 luxS mutant indicating the inherent resistance of the biofilm state. The $a g r$ and luxS quorum-sensing mutants of 1457 were similar in susceptibilities to the parent strain 1457 (within two, 2-fold dilutions of the antimicrobial) except the luxS mutant, whose $\mathrm{ED}_{75}$ for nafcillin was more than two dilutions than the parent 1457 strain (5 versus $0.625 \mu \mathrm{g} / \mathrm{mL}$ ).

Table 1. Antimicrobial susceptibility of S. epidermidis biofilms

\begin{tabular}{lcccc}
\hline \multicolumn{1}{c}{ Strains } & ATCC 55133 & 1457 & 1457 agr- & 1457 luxS- \\
\hline Farnesol $(\mathrm{mM})$ & & & & \\
$\mathrm{ED}_{50}$ & 1.25 & 2.5 & 1.25 & 0.625 \\
$\mathrm{ED}_{75}$ & 10 & 10 & 10 & 10 \\
$\mathrm{ED}_{90}$ & 20 & $>80$ & $>80$ & $>80$ \\
Nafcillin $(\mu \mathrm{g} / \mathrm{mL})$ & & & & \\
$\mathrm{ED}_{50}$ & 0.32 & 0.08 & 0.08 & 0.08 \\
$\mathrm{ED}_{75}$ & 0.625 & 0.625 & 1.25 & 5 \\
$\mathrm{ED}_{90}$ & 80 & $>80$ & $>80$ & $>80$ \\
Vancomycin $(\mu \mathrm{g} / \mathrm{L})$ & & & & \\
$\mathrm{ED}_{50}$ & 5 & 2.5 & 2.5 & 2.5 \\
$\mathrm{ED}_{75}$ & 10 & 10 & 10 & 10 \\
$\mathrm{ED}_{90}$ & 80 & $>80$ & $>80$ & $>80$ \\
\hline
\end{tabular}

Biofilm inhibitory concentrations of the antimicrobial agent at 50\%, 75\%, and $90 \%$ inhibition are reported as effective dose ${ }_{50}\left(\mathrm{ED}_{50}\right), \mathrm{ED}_{75}$, and $\mathrm{ED}_{90}$, respectively.
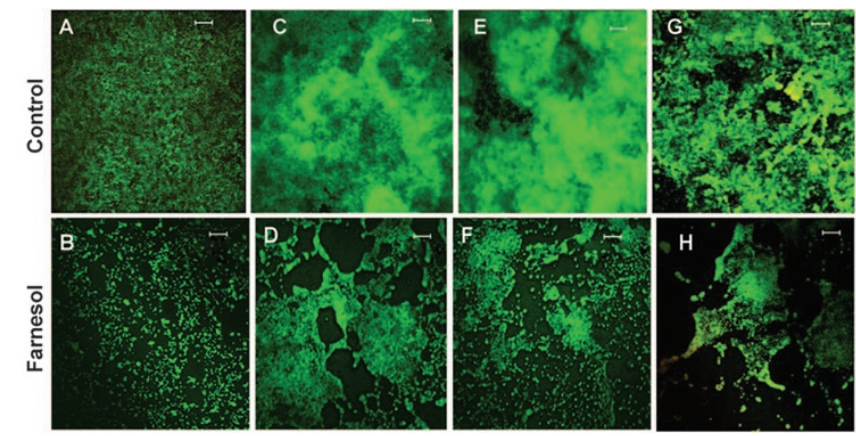

Figure 1. Biofilm evaluation by confocal microscopy. Representative images of biofilms of four strains of $S$. epidermidis exposed to DMSO (control) or FSL ( $0.5 \mathrm{mM})$ for $24 \mathrm{~h}$ : ATCC $55133(A, B), 1457(C, D), 1457$ agr mutant $(E, F)$, and 1457 luxS mutant $(G, H)$. Scale bars measure $20 \mu \mathrm{m}$. Biofilms were stained and examined by the Zeiss confocal microscope. FSL-exposed biofilms are thinner and more sparse than controls. 
In other experiments, 24-h biofilms of S. epidermidis strains were exposed to FSL at $0.5 \mathrm{mM}$ or DMSO (control). We chose $0.5 \mathrm{mM}$ of FSL, which was lower than the $\mathrm{ED}_{50}$ of all the strains to evaluate biofilm morphology. Confocal images of FSL-exposed biofilms and DMSO (control) obtained at $40 \times$ magnification were examined at $z$ intervals of $1 \mu \mathrm{m}$ (Fig. 1) and analyzed. FSL significantly decreased biovolume, substratum coverage, and mean thickness of S. epidermidis biofilms of all the four strains studied ( $p<0.05$ ) (Fig. 2).

\section{Evaluation of synergy of the antimicrobial combinations} by CIs. We evaluated FSL synergy with nafcillin and vancomycin by calculating CIs against four strains of $S$. epidermidis (Table 2). We estimated CIs at equipotency ratios (1:1 ratios of ED of FSL and the antibiotic) of $\mathrm{ED}_{50}, \mathrm{ED}_{90}$ concentrations (ATCC 55133), and $\mathrm{ED}_{75}$ ratios for 1457, $1457 \mathrm{agr}$ mutant, and 1457 luxS mutant (where $\mathrm{ED}_{90}$ measurements were not available). We also evaluated nonequipotency ratios at 1:2,
$1: 4$, and $2: 1$, where possible, from our checkerboard matrix of combinations. For FSL and vancomycin combinations, $\mathrm{ED}_{50}$ 1:1 combination ratios were the same as $\mathrm{ED}_{90} 1: 1$ for strain 55133 and same as $\mathrm{ED}_{75} 1: 1$ for strains 1457, $1457 \mathrm{agr}$ mutant, and $1457 \operatorname{luxS}$ mutant. FSL was synergistic with nafcillin and vancomycin $(\mathrm{CI}<1)$ at most equipotency and nonequipotency drug-dose ratios at $75 \%$ and $90 \%$ combination inhibitory effects of the combination $\left(\mathrm{CED}_{75}\right.$ and $\left.\mathrm{CED}_{90}\right)$ with few exceptions. The combinations that were not synergistic are given in italics in Table 2. Strains 55133 and 1457 agr mutant were synergistic at all combinations tested and strains 1457 and 1457 luxS mutant had 2 and 1 exceptions, respectively.

FSL decreased viable colony counts of $\mathrm{S}$. epidermidis in the mouse model of s.c. catheter infection. In mice after s.c. catheter infection, mice were euthanized on $\mathrm{d} 8$ of infection, catheters explanted, and cultures performed. Catheter biofilms

A

B
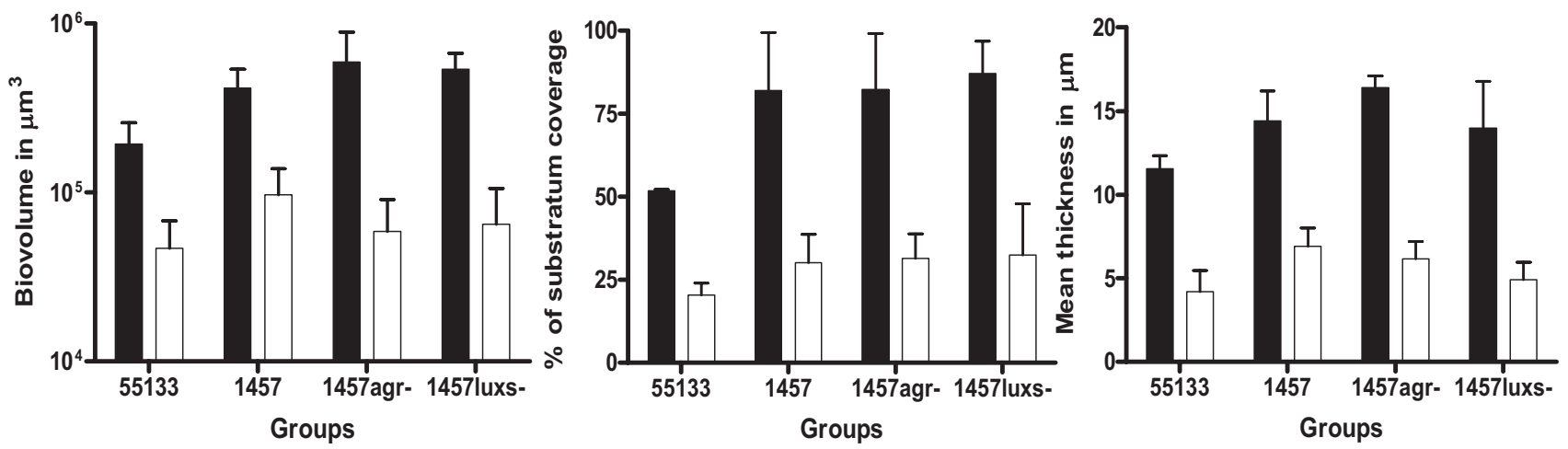

Figure 2. FSL decreases biovolume, substratum coverage, and mean thickness in biofilms in vitro. The confocal images of the 24-h biofilms exposed to DMSO or FSL were analyzed by PHLIP software, using MATLAB toolbox. The $z$-stack images were analyzed for biovolume $\left(\mu \mathrm{m}^{3}\right)(A)$, substratum coverage $(\%)(B)$, and mean thickness $(\mu \mathrm{m})(C)$. FSL $(\square)$ decreased biovolume, substratum coverage, and mean thickness significantly $(p<0.05)$, when compared with DMSO control (匹) for all four S. epidermidis strains.

Table 2. CIs for farnesol-antibiotic combinations

\begin{tabular}{|c|c|c|c|c|c|}
\hline \multirow{2}{*}{$\begin{array}{c}\text { Ratios } \\
\text { FSL: NAF }\end{array}$} & \multicolumn{2}{|c|}{$\mathrm{CI}$ values in mean (SD) } & \multirow{2}{*}{$\begin{array}{c}\text { Ratios } \\
\text { FSL:VAN }\end{array}$} & \multicolumn{2}{|c|}{$\mathrm{CI}$ values in mean (SD) } \\
\hline & $\mathrm{CED}_{75}$ & $\mathrm{CED}_{90}$ & & $\mathrm{CED}_{75}$ & $\mathrm{CED}_{90}$ \\
\hline \multicolumn{6}{|l|}{ ATCC 55133} \\
\hline $\mathrm{ED}_{50} 1: 1$ & $0(0)$ & $0.26(0.45)$ & $\mathrm{ED}_{50} 1: 1=\mathrm{ED}_{90} 1: 1$ & $0.13(0.11)$ & $0.1(0.02)$ \\
\hline $\mathrm{ED}_{90} 1: 1$ & $0.5(0.5)$ & $0.04(0.01)$ & $\mathrm{ED}_{90} 1: 2$ & $0.10(0.07)$ & $0.07(0.02)$ \\
\hline $\mathrm{ED}_{90} 1: 2$ & $0.13(0.11)$ & $0.06(0.01)$ & $\mathrm{ED}_{90} 2: 1$ & $0.18(0.17)$ & $0.1(0.04)$ \\
\hline $\mathrm{ED}_{90} 2: 1$ & $0.22(0.19)$ & $0.07(0.03)$ & & & \\
\hline \multicolumn{6}{|l|}{1457} \\
\hline $\mathrm{ED}_{75} 1: 1$ & $3.82(0.53)$ & $0.05(0)$ & $\mathrm{ED}_{50} 1: 1=\mathrm{ED}_{75} 1: 1$ & $0.24(0.05)$ & $0.08(0.02)$ \\
\hline $\mathrm{ED}_{75} 1: 2$ & $2.41(1.80)$ & $0.21(0.13)$ & $\mathrm{ED}_{75} 1: 2$ & $0.29(0.09)$ & $0.15(0.03)$ \\
\hline $\mathrm{ED}_{75} 1: 4$ & $0.46(0.53)$ & $0.34(0.13)$ & $\mathrm{ED}_{75} 2: 1$ & $0.21(0.03)$ & $0.07(0.04)$ \\
\hline \multicolumn{6}{|l|}{1457 agr- } \\
\hline $\mathrm{ED}_{75} 1: 1$ & $0.69(0.26)$ & $0.41(0.35)$ & $\mathrm{ED}_{50} 1: 1=\mathrm{ED}_{75} 1: 1$ & $0.30(0.29)$ & $0.2(0.11)$ \\
\hline $\mathrm{ED}_{75} 1: 2$ & $0.55(0.51)$ & $0.59(0.13)$ & $\mathrm{ED}_{75} 1: 2$ & $0.33(0.28)$ & $0.35(0.16)$ \\
\hline $\mathrm{ED}_{75} 1: 4$ & $0.48(0.42)$ & $0.9(0.79)$ & $\mathrm{ED}_{75} 2: 1$ & $0.26(0.25)$ & $0.14(0.06)$ \\
\hline \multicolumn{6}{|l|}{1457 luxS- } \\
\hline $\mathrm{ED}_{75} 1: 1$ & $0.21(0.30)$ & $0.03(0.02)$ & $\mathrm{ED}_{50} 1: 1=\mathrm{ED}_{75} 1: 1$ & $0.27(0.06)$ & $0.05(0.02)$ \\
\hline $\mathrm{ED}_{75} 1: 2$ & $0.01(0.02)$ & $0.09(0.07)$ & $\mathrm{ED}_{75} 1: 2$ & $0.28(0.06)$ & $0.06(0.024)$ \\
\hline $\mathrm{ED}_{75} 1: 4$ & $0.08(0.07)$ & $0.12(0.09)$ & $\mathrm{ED}_{75} 2: 1$ & $0.25(0.1)$ & $0.05(0.02)$ \\
\hline $\mathrm{ED}_{50} 1: 1$ & $0.29(0.27)$ & $10.57(182)$ & $\mathrm{ED}_{50} 1: 1$ & $0.10(0.03)$ & $0.03(0.014)$ \\
\hline
\end{tabular}

FSL, farnesol; NAF, nafcillin; VAN, vancomycin. $\mathrm{ED}_{50}, \mathrm{ED}_{75}$, and $\mathrm{ED}_{90}$ represent $50 \%, 75 \%$, and $90 \%$ inhibitory effects of the individual antimicrobial agent, respectively. $\mathrm{CED}_{75}$ and $\mathrm{CED}_{90}$ represent $75 \%$ and $90 \%$ inhibitory effects of farnesol-antibiotic combination, respectively. CI derived by the median effects principle, indicate synergy if $\mathrm{CI}<1$, additive effect if $\mathrm{CI}=1$ and antagonism if $\mathrm{CI}>1$. Nonsynergistic combinations are italicized. 

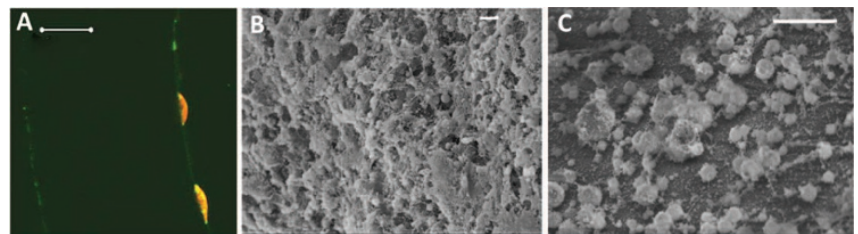

Figure 3. FSL decreases catheter biofilms. In 3-wk-old FVB mice, 1.5-inch Teflon catheter segments were inserted s.c. Catheters were preincubated in $S$. epidermidis suspension for $2 \mathrm{~h}$ before insertion to aid biofilm development. The catheter segments were explanted on d 8 of implantation, sectioned into thin slices, stained with LIVE/DEAD stain, and examined under the Zeiss confocal microscope $(A)$. Scale bar measures $50 \mu \mathrm{m}$ in $(A)$. Some catheter segments were examined by scanning electron microscopy $(B, C)$. Confocal imaging and electron microscopy confirmed $S$. epidermidis biofilm formation $(A, B)$. FSL treatment $(100 \mu \mathrm{g} / \mathrm{g}$, s.c. for $6 \mathrm{~d})$ decreased S. epidermidis catheter biofilms $(C)$. Scale bars measure $10 \mu \mathrm{m}$ in $(B)$ and $(C)$.
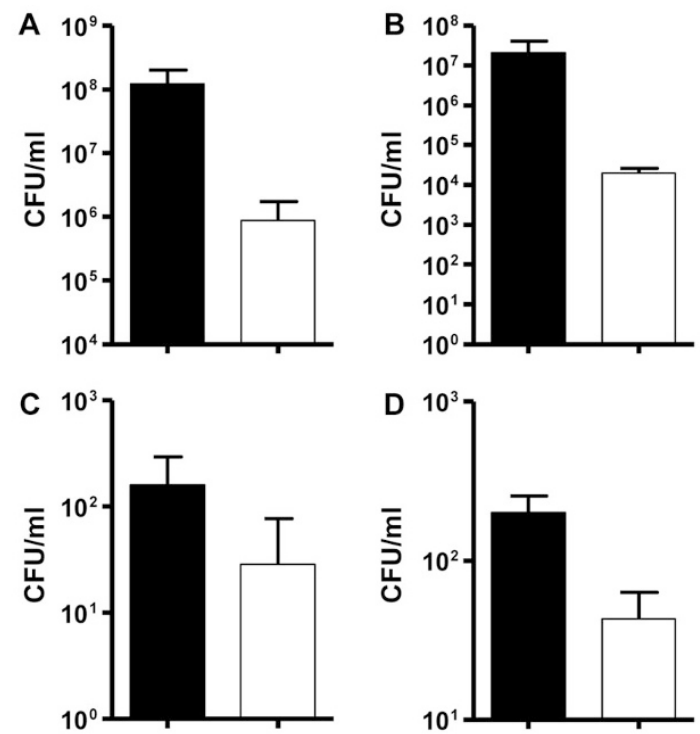

Figure 4. FSL decreases viable cell counts of S. epidermidis during catheter infection in mice. Mice were euthanized on d 8 of catheter implantation and cultures of the catheters $(A)$ and skin and tissue around the catheter (pericatheter) $(B)$ were performed. Systemic dissemination was assessed by cultures of blood $(C)$ and kidney homogenate (kidneys of one animal homogenized in 1 $\mathrm{mL}$ of PBS) $(C)$. FSL treatment $(n=7)$ was compared with DMSO control $(n=5)$. FSL treatment significantly decreased catheter $\mathrm{CFU} / \mathrm{mL}$, pericatheter infection, and systemic dissemination $(p<0.05)$. All panels: DMSO control $(\square)$, FSL treatment $(\square)$.

were confirmed by confocal and electron microscopy (Fig. 3A, $B$ ). FSL decreased catheter biofilms (Fig. 3C) compared with controls (Fig. 3B). FSL treatment decreased catheter and pericatheter infection of $S$. epidermidis as shown by significantly decreased viable colony counts $(\mathrm{CFU} / \mathrm{mL})$ from catheter (Fig. 4A) and pericatheter tissues (Fig. 4B). FSL treatment also decreased systemic dissemination of $S$. epidermidis as shown by decreased blood and kidney CFU/mL (Fig. 4C, D). There were not significant differences in weight gain, activity, or local reactions in FSL-treated mice compared with controls (data not shown).

FSL inhibits biofilms of bioluminescent S. epidermidis $X e n 43$ in vitro and in vivo. As an alternative approach to assess the effects of FSL on S. epidermidis biofilms in vivo, a bioluminescent strain was used. The utility of this strain was
A
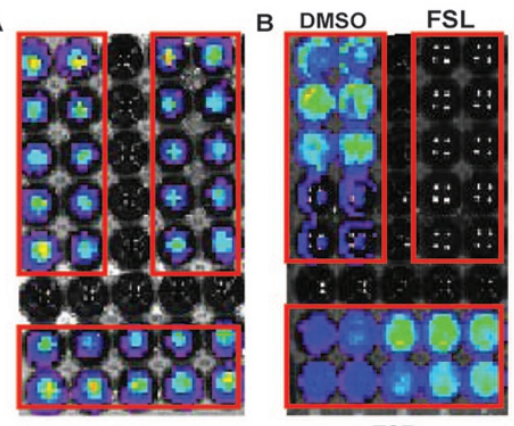

C

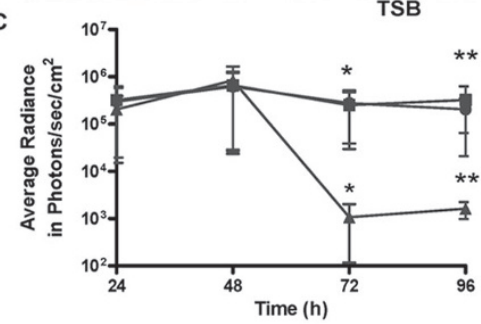

Figure 5. Biofilm bioluminescence of Xen 43 decreased by FSL. Xen 43 biofilms were developed in 30 wells of an opaque 96-well microtiter plate $(A)$. At $48 \mathrm{~h}$, supernatants were removed and unadhered cells were washed with PBS. Three groups of 10 wells each were exposed to DMSO, FSL $(0.5 \mathrm{mM})$, or TSB media for $24 \mathrm{~h}(B)$. Bioluminescence was monitored at 24, 48, 72, and $96 \mathrm{~h}$ and compared among the three exposures. FSL exposure significantly reduced average radiance compared with DMSO- or TSB-exposed wells $(*$ and $* * p<0.05)(C)$. $\mathbf{0}$ : DMSO; $\bullet$ : TSB; $\mathbf{\Delta}:$ FSL.

validated in vitro. After $48 \mathrm{~h}$, Xen 43 biofilms were divided into three groups of 10 wells and treated with FSL, DMSO, or fresh medium. In vitro, Xen 43 biofilms, bioluminescence was not significantly different among the three groups of 10 wells at $48 \mathrm{~h}$, before exposure to FSL (Fig. 5A). After exposure to DMSO, FSL (FSL) $(0.5 \mathrm{mM})$, or TSB for $24 \mathrm{~h}$, FSL significantly reduced bioluminescence compared with DMSO or TSB (Fig. 5B, C). Bioluminescence did not differ significantly between DMSO- and TSB-exposed biofilms.

In vivo, s.c. catheter biofilm infection of Xen 43, bioluminescence over the infected catheters was monitored daily for $5 \mathrm{~d}$ in live animals (Fig. 6A, B). Average radiance was similar on d 1 and d 2, before exposure to FSL. After FSL treatment, a significant decrease in bioluminescence was observed on $\mathrm{d}$ 3,4 , and 5 of infection. FSL treatment significantly decreased biofilm infection in vivo.

\section{DISCUSSION}

FSL inhibited biofilms of S. epidermidis both in vitro and in vivo and was synergistic with nafcillin and vancomycin at most combination ratios. In our model of s.c. catheter infection in mice which is clinically relevant, FSL treatment decreased catheter infection and systemic dissemination. We also confirmed the biofilm inhibiting effects of FSL in real time, using a bioluminescent strain of $S$. epidermidis.

We report $\mathrm{ED}_{50}, \mathrm{ED}_{75}$, and $\mathrm{ED}_{90}$ of $\mathrm{FSL}$, nafcillin, and vancomycin against biofilms of two clinical isolates and three laboratory strains of S. epidermidis, all of which were sensitive to nafcillin (minimum inhibitory concentration ${ }_{50}$ $\left.\left[\mathrm{MIC}_{50}\right]\right)<0.5 \mu \mathrm{g} / \mathrm{mL}$ ) and vancomycin $\left(\mathrm{MIC}_{50}<2 \mu \mathrm{g} / \mathrm{mL}\right.$ ) in the planktonic state (data not shown). We evaluated quo- 
A
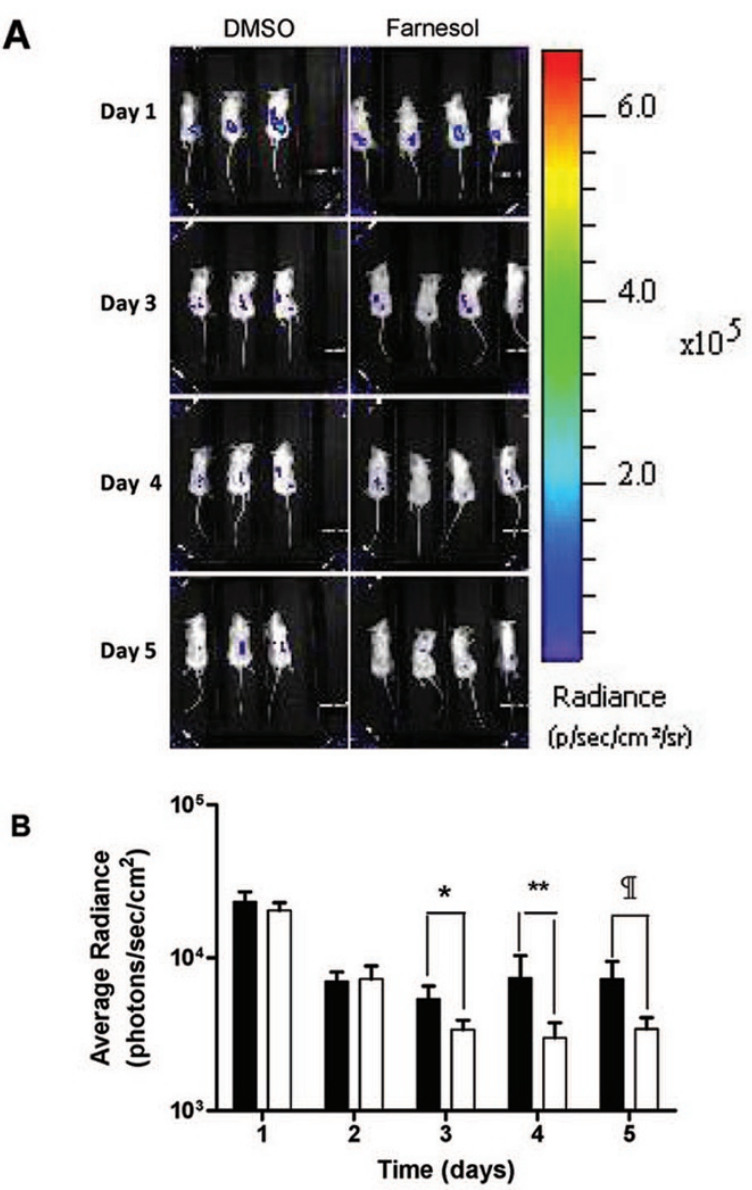

Figure 6. FSL decreases bioluminescence in S. epidermidis catheter infection in vivo. A bioluminescent strain of $S$. epidermidis 1457 Xen 43 was used in the s.c. catheter infection model in mice and bioluminescence followed serially for $5 \mathrm{~d}(A)$. Mice were treated with FSL at $100 \mu \mathrm{g} / \mathrm{g} / \mathrm{d}$ or DMSO (control) from $\mathrm{d} 2$ of infection for $4 \mathrm{~d}$. Bioluminescence was quantified and expressed as average radiance in photons $/ \mathrm{s} / \mathrm{cm}^{2}(B)$. FSL treatment $(\square)$ decreased bioluminescence significantly $(*, * *$, and $\mathbb{T} p<0.05)$ on $\mathrm{d} 3,4$, and 5 compared with DMSO control (ם).

rum-sensing mutants (agr and luxS mutants) of S. epidermidis 1457 , as these mutant strains form thicker biofilms than WT strains and spontaneous agr mutants predominate in chronic biofilm infections (11). Quorum-sensing mechanisms determine antibiotic and biocide susceptibility in Pseudomonas aeruginosa and we sought to clarify FSL susceptibility of these quorum-sensing mutants in S. epidermidis (19). The agr and $\operatorname{luxS}$ quorum-sensing mutants were similar in susceptibilities to the parent strain 1457 (within two, 2-fold dilutions of the antimicrobial) except the luxS mutant, whose $\mathrm{ED}_{75}$ for nafcillin was more than two dilutions than the parent strain (5 versus $0.625 \mu \mathrm{g} / \mathrm{mL}$ ).

Gomes et al. (20) reported the antibacterial effects of FSL on planktonic cells of $S$. epidermidis at concentrations up to $300 \mu \mathrm{M}$ and reported S. epidermidis susceptibility to FSL at $100 \mu \mathrm{M}$. However, biofilms were tolerant to FSL in vitro. Gomes et al. did not report MICs or EDs performed according to standardized guidelines or the effects of FSL on biofilms in vivo. Antibacterial effects of FSL on S. aureus at $150 \mu \mathrm{M}$ concentration and synergy with gentamicin at $100 \mu \mathrm{M}$ on $S$. aureus biofilms have been reported (10). Possible mechanisms for the antibacterial effects of FSL have been explored. FSL at $100 \mu \mathrm{g} / \mathrm{mL}$ inhibits the procoagulant effect, production of $S$. aureus exotoxins, and potentiated the effects of cell wallacting ampicillin (21). Inoue et al. (22) demonstrated that $\mathrm{K}^{+}$ ion leakage from $S$. aureus induced by the terpene alcohols such as FSL correlated with the antibacterial effects. Thus, the antibacterial effects of FSL may be due to its effects on membrane integrity.

We evaluated the morphology of S. epidermidis biofilms exposed to FSL at $0.5 \mathrm{mM}$ and observed a significant inhibiting effect. Detailed evaluation on confocal imaging revealed significant decrease in biovolume, mean thickness, and substratum coverage of FSL-exposed biofilms of both WT and quorum-sensing mutants. The efficacy of FSL at concentrations much lower than the estimated $\mathrm{ED}_{50}(0.625$ to $2.5 \mathrm{mM})$ against $S$. epidermidis biofilms may have clinical implications in the treatment of biofilm-related catheter and deviceassociated healthcare infections.

Biofilms are inherently resistant to antibiotics, and antimicrobial combinations may be an important strategy against biofilm infections. Antimicrobial combinations against biofilms may enhance efficacy, reduce drug dosages, and minimize the development of drug resistance. Therefore, we evaluated combinations of FSL with the commonly used antistaphylococcal antibiotics; nafcillin, and vancomycin, by discerning inhibitory end points by the XTT assay. We used the median effects principle expounded by Chou (17) to evaluate synergy for the antimicrobial combinations. Evaluation of antimicrobial combinations by the median effects method is widely used in cancer and infectious diseases research (17). Advantages of this method include surmounting the assumption that drug interactions are linear across drug dosages and effects. No general equation fits all the doseresponse curves because mechanisms of drug actions differ. Dose-response curves evaluated at various dose effects may overcome this problem. Therefore, we evaluated drug combinations in a systematic manner at two different dose effects, $\mathrm{CED}_{75}$ and $\mathrm{CED}_{90}$, at constant drug ratios, including equipotency ratios of the drug combinations. We observed synergy at most combination ratios with few exceptions.

We also evaluated the effects of FSL in vivo in a mouse model of s.c. catheter infection that is a clinically relevant model of device-associated infection. We confirmed the formation of biofilms on the s.c. implanted catheters in mice by electron and confocal laser microscopy. FSL treatment significantly decreased catheter infection and systemic dissemination. The dose of FSL was extrapolated from animal studies and that was higher than our estimated $\mathrm{ED}_{50}$ in vitro (18). Other investigators have evaluated higher doses of FSL $(1 \mathrm{~mL}$ of $20 \mathrm{mM}$ i.p.) in systemic candidiasis in mice $(23,24)$. FSL treatment by injections once a day at $100 \mu \mathrm{g} / \mathrm{g}$ did not cause any local or systemic adverse effects in mice. We are not aware of other studies evaluating the antibiofilm effects of FSL on S. epidermidis biofilms in vivo.

Real-time photonic imaging of animals offers advantages over conventional animal infection models. Real-time monitoring has the advantage of serial monitoring of infection and 
may decrease the sample size of animals (25). Growth curves of Xen 43 are similar to the parent strain 1457, and the bioluminescence is directly proportional to the $\mathrm{CFU} / \mathrm{mL}$ (data not shown). The feasibility of monitoring catheter infection in mice using a bioluminescent strain of S. epidermidis Xen 43 has been reported (12). We evaluated the effects of FSL on biofilms in vitro and on s.c. catheter infection using the bioluminescent strain of $S$. epidermidis Xen43, in vivo. FSL significantly decreased Xen 43 biofilm bioluminescence in vitro and in our catheter infection model in vivo.

Standard guidelines for biofilm antimicrobial susceptibility do not exist and we adapted CLSI guidelines for planktonic cells to evaluate EDs against biofilms. We evaluated FSL (0.5 $\mathrm{mM}$ ) on biofilms formed in vitro and a daily dose of $100 \mu \mathrm{g} / \mathrm{g}$ s.c. for $6 \mathrm{~d}$ in the animal model. It is possible that several other concentrations or doses are effective and the optimum effective dose that has clinical applicability needs to be established. The mouse model of s.c. catheter infection mimics a foreign device infection, and to evaluate the effects on intravascular catheter biofilm infection, our future experiments will focus on a vascular indwelling catheter infection in mice.

We have evaluated and confirmed the efficacy of FSL against biofilms of $S$. epidermidis by multiple approaches, both in vitro and in a clinically relevant s.c. catheter biofilm infection in mice in vivo. No adverse effects of FSL treatment were observed in this animal model. We also observed synergy of FSL with nafcillin and vancomycin. We conclude that FSL alone or in combination with nafcillin or vancomycin may be effective against device-associated biofilm infections and improve clinical outcomes.

Acknowledgments. We appreciate the advice and help of Waleed Gaber, Ph.D., at the small animal imaging facility at Texas Children's Hospital. We thank the productive discussions with Paul Fey, Ph.D., and Deborah Hogan, Ph.D. We also thank all members of the Versalovic lab for their insightful discussion and critiques.

\section{REFERENCES}

1. Fey PD 2010 Modality of bacterial growth presents unique targets: how do we treat biofilm-mediated infections? Curr Opin Microbiol 13:610-615

2. Potera C 1999 Forging a link between biofilms and disease. Science 283:1837-1839

3. Raad I, Hanna H, Maki D 2007 Intravascular catheter-related infections: advances in diagnosis, prevention, and management. Lancet Infect Dis 7:645-657

4. Fey PD, Olson ME 2010 Current concepts in biofilm formation of Staphylococcus epidermidis. Future Microbiol 5:917-933

5. Stoll BJ, Hansen N, Fanaroff AA, Wright LL, Carlo WA, Ehrenkranz RA, Lemons JA, Donovan EF, Stark AR, Tyson JE, Oh W, Bauer CR, Korones SB, Shankaran S, Laptook AR, Stevenson DK, Papile LA, Poole WK 2002 Late-onset sepsis in very low birth weight neonates: the experience of the NICHD Neonatal Research Network. Pediatrics 110:285-291

6. Adams-Chapman I, Stoll BJ 2006 Neonatal infection and long-term neurodevelopmental outcome in the preterm infant. Curr Opin Infect Dis 19:290-297

7. Hornby JM, Jensen EC, Lisec AD, Tasto JJ, Jahnke B, Shoemaker R, Dussault P, Nickerson KW 2001 Quorum sensing in the dimorphic fungus Candida albicans is mediated by farnesol. Appl Environ Microbiol 67:2982-2992

8. Weber K, Sohr R, Schulz B, Fleischhacker M, Ruhnke M 2008 Secretion of E,E-farnesol and biofilm formation in eight different Candida species. Antimicrob Agents Chemother 52:1859-1861

9. Ramage G, Saville SP, Wickes BL, Lopez-Ribot JL 2002 Inhibition of Candida albicans biofilm formation by farnesol, a quorum-sensing molecule. Appl Environ Microbiol 68:5459-5463

10. Jabra-Rizk MA, Meiller TF, James CE, Shirtliff ME 2006 Effect of farnesol on Staphylococcus aureus biofilm formation and antimicrobial susceptibility. Antimicrob Agents Chemother 50:1463-1469

11. Vuong C, Kocianova S, Yao Y, Carmody AB, Otto M 2004 Increased colonization of indwelling medical devices by quorum-sensing mutants of Staphylococcus epidermidis in vivo. J Infect Dis 190:1498-1505

12. Vuong C, Kocianova S, Yu J, Kadurugamuwa JL, Otto M 2008 Development of real-time in vivo imaging of device-related Staphylococcus epidermidis infection in mice and influence of animal immune status on susceptibility to infection. J Infect Dis 198:258-261

13. Wikler MA, Clinical and Laboratory Standards Institute 2009 Methods for dilution antimicrobial susceptibility tests for bacteria that grow aerobically; approved standard, eighth edition. CLSI document M07-A8. Clinical and Laboratory Standards Institute, Wayne. Available at: http://www.clsi.org/source/orders/free/m07-a8.pdf (Accessed, June 10, 2011)

14. Hawser SP, Norris H, Jessup CJ, Ghannoum MA 1998 Comparison of a 2,3-bis(2methoxy-4-nitro-5-sulfophenyl)-5-[(phenylamino)carbonyl]-2H-t etrazolium hydroxide (XTT) colorimetric method with the standardized National Committee for Clinical Laboratory Standards method of testing clinical yeast isolates for susceptibility to antifungal agents. J Clin Microbiol 36:1450-1452

15. Cerca N, Martins S, Cerca F, Jefferson KK, Pier GB, Oliveira R, Azeredo J 2005 Comparative assessment of antibiotic susceptibility of coagulase-negative staphylococci in biofilm versus planktonic culture as assessed by bacterial enumeration or rapid XTT colorimetry. J Antimicrob Chemother 56:331-336

16. Mueller LN, de Brouwer JFC, Almeida JS, Stal LJ, Xavier JB 2006 Analysis of a marine phototrophic biofilm by confocal laser scanning microscopy using the new image quantification software PHLIP. BMC Ecol 6:1

17. Chou TC 2006 Theoretical basis, experimental design, and computerized simulation of synergism and antagonism in drug combination studies. Pharmacol Rev 58:621681

18. Chaudhary SC, Alam MS, Siddiqui MS, Athar M 2009 Chemopreventive effect of farnesol on DMBA/TPA-induced skin tumorigenesis: involvement of inflammation, Ras-ERK pathway and apoptosis. Life Sci 85:196-205

19. Bjarnsholt T, Jensen PO, Burmolle M, Hentzer M, Haagensen JA, Hougen HP, Calum H, Madsen KG, Moser C, Molin S, Hoiby N, Givskov M 2005 Pseudomonas aeruginosa tolerance to tobramycin, hydrogen peroxide and polymorphonuclear leukocytes is quorum-sensing dependent. Microbiology 151:373-383

20. Gomes FI, Teixeira P, Azeredo J, Oliveira R 2009 Effect of farnesol on planktonic and biofilm cells of Staphylococcus epidermidis. Curr Microbiol 59:118-122

21. Akiyama H, Oono T, Huh WK, Yamasaki O, Ogawa S, Katsuyama M, Ichikawa H, Iwatsuki K 2002 Actions of farnesol and xylitol against Staphylococcus aureus. Chemotherapy 48:122-128

22. Inoue Y, Shiraishi A, Hada T, Hirose K, Hamashima H, Shimada J 2004 The antibacterial effects of terpene alcohols on Staphylococcus aureus and their mode of action. FEMS Microbiol Lett 237:325-331

23. Navarathna DH, Nickerson KW, Duhamel GE, Jerrels TR, Petro TM 2007 Exogenous farnesol interferes with the normal progression of cytokine expression during candidiasis in a mouse model. Infect Immun 75:4006-4011

24. Navarathna DH, Hornby JM, Krishnan N, Parkhurst A, Duhamel GE, Nickerson KW 2007 Effect of farnesol on a mouse model of systemic candidiasis, determined by use of a DPP3 knockout mutant of Candida albicans. Infect Immun 75:1609-1618

25. Francis KP, Yu J, Bellinger-Kawahara C, Joh D, Hawkinson MJ, Xiao G, Purchio TF, Caparon MG, Lipsitch M, Contag PR 2001 Visualizing pneumococcal infections in the lungs of live mice using bioluminescent Streptococcus pneumoniae transformed with a novel gram-positive lux transposon. Infect Immun 69:3350-3358 